\title{
A rare entity of Primary Ewing sarcoma in kidney
}

\author{
Li Cheng ${ }^{1}$, Yujie Xu ${ }^{1}$, Hong Song ${ }^{2}$, Houbao Huang ${ }^{1}$ and Dong Zhuo ${ }^{1 *}$
}

\begin{abstract}
Background: Ewing sarcoma (ES) or primitive neuroectodermal tumors (PNET) represents a spectrum of poorly differentiated and aggressive malignancies. It rarely arises from the kidney and accounts for less than $1 \%$ of renal mass. Given the uncharacteristic clinical symptoms and imaging features, renal Ewing sarcoma (RES) is often diagnosed by postoperative pathology.

Case presentation: Herein, we depicted a case of RES, which was administrated in our institution by chief complaints of intermittent left plank pain and palpable abdominal mass. We demonstrated the aggressive behavior of this renal malignancy and summarized its therapeutic modalities and outcomes.

Conclusion: The diagnosis of RES relies on integrated analysis including histomorphology, immunohistochemical staining and confirmation of molecular-genetic testing. Despite the surgery and adjuvant therapy, optimized and potent therapeutic regimes are still urgently needed to improve the poor prognosis of RES.
\end{abstract}

Keywords: Renal ewing sarcoma (RES), Primitive neuroectodermal tumor (PNET), Nephrectomy, Chemotherapy

\section{Background}

Ewing sarcoma (ES), also known as primitive neuroectodermal tumors (PNET), is a group of undifferentiated tumors that originates from neuroectoderm. It typically encountered in the bone and soft tissue of children and young adults [1]. The occurrence of ES/PNET in kidney is firstly depicted in 1975 [2]. Up to date, there emerges limited reported cases of ES/PNET in kidney by the rarity of this disease. Renal Ewing sarcoma (RES) presents highly malignant, grows rapidly, and metastases early to the lung, bone and lymph node $[3,4]$. Thus, it is indispensable to distinguish RES from other renal entities. Herein, we present a case of an adult patient with RES who was diagnosed and administered in our hospital. We report this case in accordance with the CARE-Guideline [5].

\footnotetext{
*Correspondence: zhuodongwh@163.com

1 Department of Urology, Anhui Province, The First Affiliated Hospital

of Wannan Medical College, Wuhu, People's Republic of China

Full list of author information is available at the end of the article
}

\section{Case presentation}

A 31-year-old female patient was admitted with chief complaints of intermittent pain in the left plank and palpable abdominal mass. No urinary symptoms such as haematuria and dysuria were found. Her past medical history and family history of malignancy were unremarkable. Results of routine blood and urine examination as well as tumor markers including AFP, AFP-L3, CEA, CA199, CA125 and SCC were within the normal range. Enhanced computed tomography (CT) demonstrated a hypoechoic mass $(18 \mathrm{~cm} \times 14.5 \mathrm{~cm} \times 14 \mathrm{~cm})$ from the left renal with central necrosis, no invasion to renal veins or inferior vena cava (IVC) was reported (Fig. 1a, b). Further clinical investigations including chest HRCT showed no evidence of metastasis. 3-dimensional imaging of the neoplasm was conducted (Fig. 1c), and an open, left radical nephrectomy was performed. Macroscopic examination showed a huge soft tissue mass attached to the spleen and pancreas tail. In surgery, the spleen was separated from the tumor capsule, and partial left adrenal gland and distal pancreatic involving 


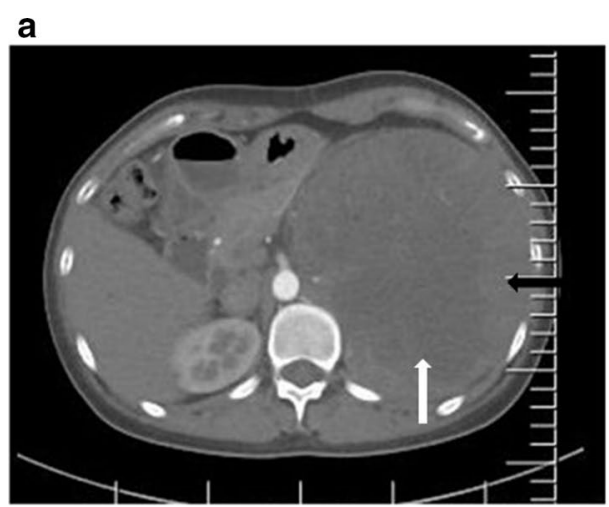

b

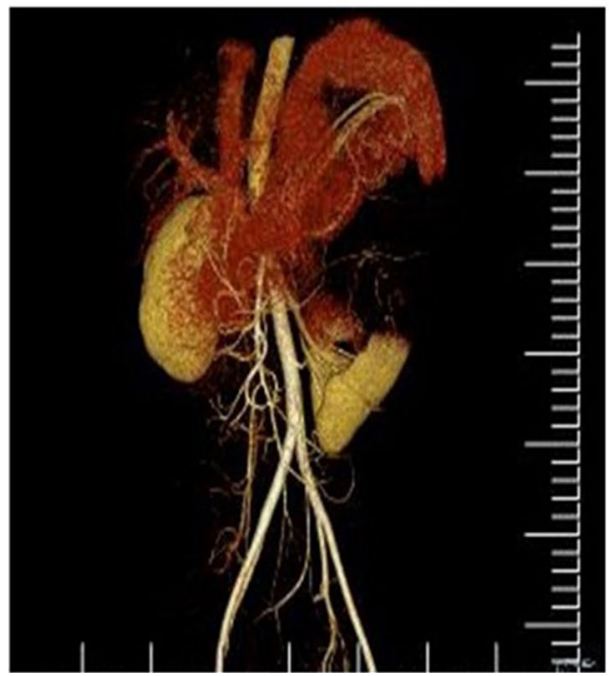

C

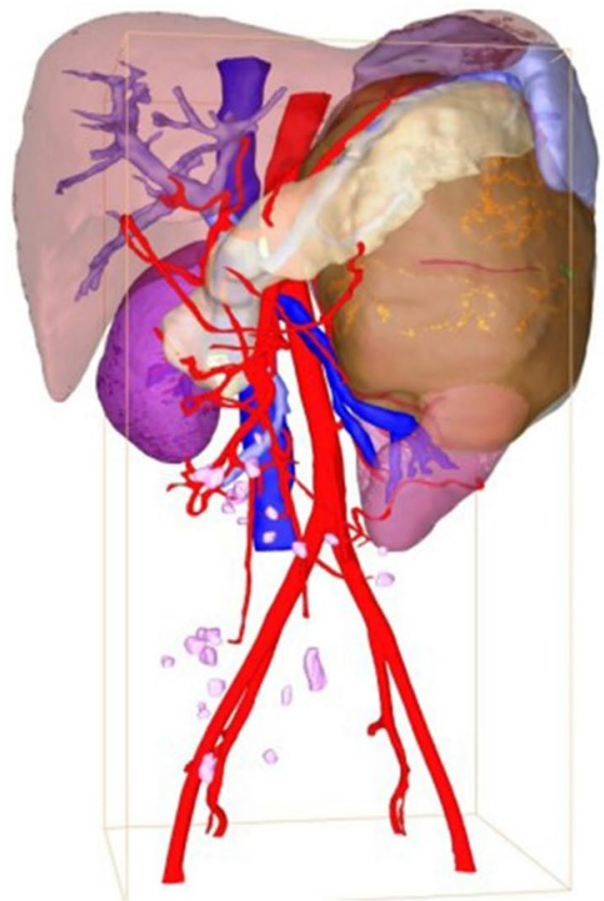

Fig. 1 CT scan and three-dimensional imaging. Enhanced computed tomography (CT) revealed a hypoechoic mass about $18 \mathrm{~cm} \times 14.5 \mathrm{~cm} \times 14 \mathrm{~cm}$ arising from the left renal. a Cross-section. The black arrows represent the enhanced solid tumors and the white arrows represent non-enhanced tumor necrosis components. b Angiography of bilateral renal. c Three-dimensional imaging of the neoplasm

about $2 \mathrm{~cm} \times 1.5 \mathrm{~cm} \times 1 \mathrm{~cm}$ pancreas tail tissue were excised with the assistance of hepatobiliary surgeons. Microscopic examination revealed a small round cell tumor with focal necrosis, endovascular tumor thrombi and adrenal involvement were detected (Fig. 2a, b). The tumor cells were arranged in sheet with fiber segmentation. Immunohistochemistry results indicated positive stained for AE1/AE3, CD99, CD56, synaptophysin (Syn), Ki-67(50\%), and negatively stained for S-100, EMA, CgA, WT1, Desmin, SMA and MyoD1 (Fig. 2c-g). In the light of these findings, a pathological diagnosis of primary ES/PNET of the left renal was made. The diagnosis was further confirmed by fluorescence in situ hybridization (FISH) by detecting the rearrangements of $t$ (22q12): EWS-FLI1 type 1 translocation (Fig. 2h). A PET-CT examination was conducted at 3 months postoperatively and showed no recurrence or metastasis. Adjuvant chemotherapy including vincristine, doxorubicin, cyclophosphamide (VAC), ifosfamide, etoposide (IE) and carboplatin $(\mathrm{AUC}=4)$ was delivered after surgery. During the triweekly chemotherapy, metastasis was found in the retroperitoneal lymph node at 6 months postoperative, followed by multiple metastases to the left neck, bilateral adrenal gland, psoas major, retroperitoneum and bilateral diaphragm angles. The chemotherapy was maintained by the follow-up at 12 months postoperatively, and lung metastasis was detected by PET-CT scan. Afterwards, the antiangiogenic drug, apatinib, was recommended by the oncologist due to the intolerance to chemotherapy. This patient survived with tumor by the latest follow-up at 18 months postoperatively. 

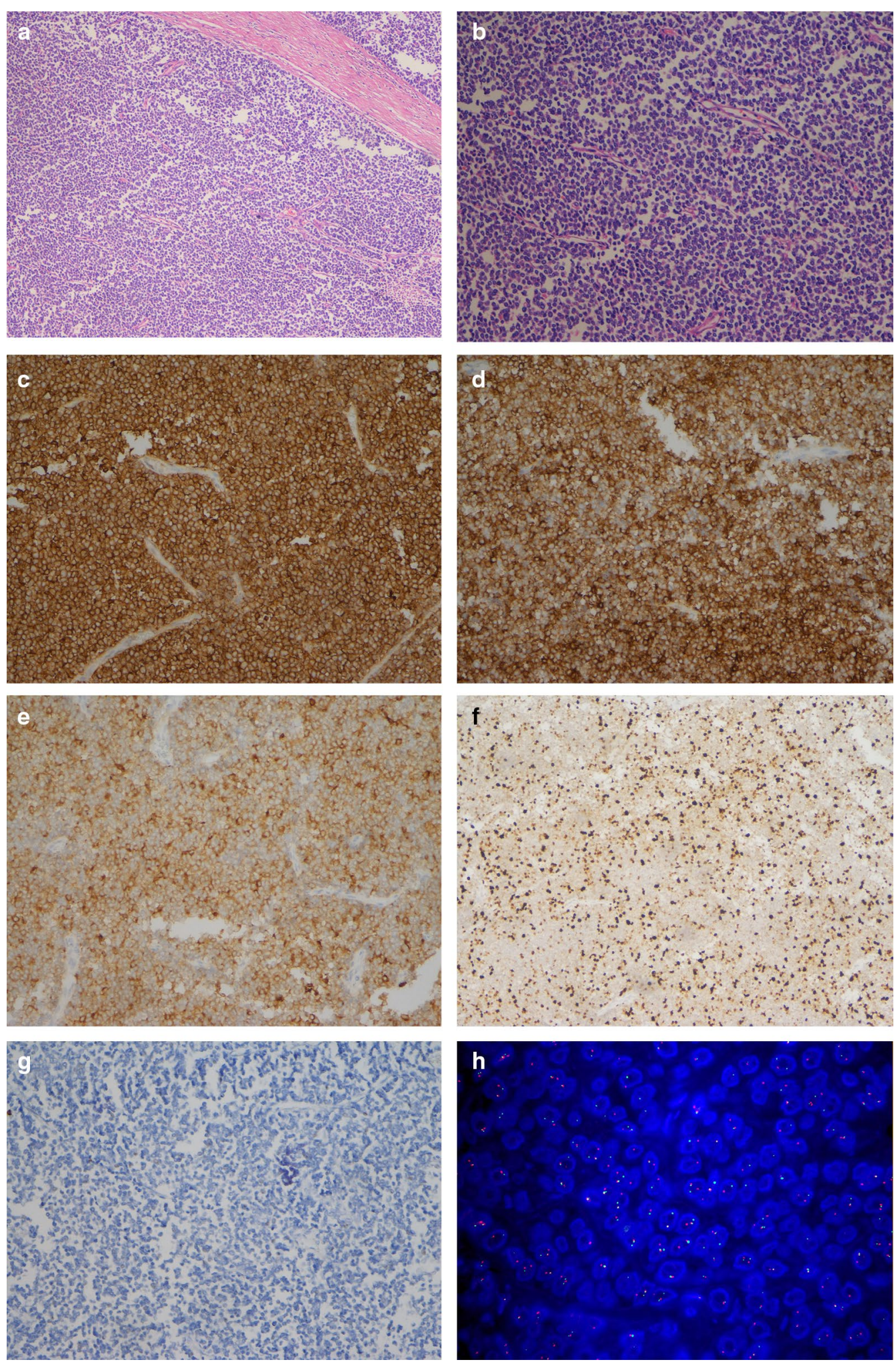

Fig. 2 Pathological examination of the surgical specimen. HE staining (a and $\mathbf{b})$ indicated that the tumor was composed of a population of small round cells arranged in sheet. Immunohistochemical staining was positive for c CD99, d CD56, e syn and f Ki67 (50\%) and negative for $\mathbf{g}$ S100. Magnification details: $\mathbf{a} \times 100 ; \mathbf{b} \times 200 ; \mathbf{c} \times 100 ; \mathbf{d} \times 100 ; \mathbf{e} \times 200 ; \mathbf{f} \times 100 ; \mathbf{g} \times 100 . \mathbf{g}$ EWSR1 gene translocation was detected by fluorescence in situ hybridization testing (FISH) 


\section{Discussion}

ES rarely presents as a primary kidney tumor. The clinical symptoms and signs are usually nonspecific, such as abdominal or flank pain, hematuria and palpable mass. Imaging examinations often reveal indistinctive signs but central necrosis and hemorrhage, which raises the difficulty of accurate preoperative diagnosis of RES. Mostly, the diagnosis was made by postoperative pathological results. Histologically, RES is composed of uniform small round cells. The major differential diagnosis for this histomorphology in kidney consists of Wilms tumor, malignant lymphoma, synovial sarcoma, solid variant of alveolar rhabdomyosarcoma, clear cell sarcoma of the kidney, small-cell neuroendocrine carcinoma, desmoplastic small round blue cell tumor and small cell carcinoma [6]. These poorly differentiated small round cell tumors share with overlapping morphologic features but have varied prognosis, which makes the distinguish diagnosis notoriously difficult but imperative. Advances in immunohistochemistry and molecular-genetic techniques are helpful, as numerous positive and negative immunohistochemical markers offer a better distinction. CD99 and FLI-1 were commonly positive in ES/PNET, including those arising from the kidney. CD45, WT-1 and Desmin have been reported as negative $[7,8]$. These markers by immunohistochemical staining established a useful albeit imprecise diagnosis panel of RES, as some of the markers are shared among small round blue tumors. For the indefinable cases, the ancillary molecular testing is recommended $[9,10]$. ES/PNET is characterized by fixed chromosomal translocation $t$ (11:22) between the genes EWS (22q12) and FLI-1 (11q24). In the present case, the EWS-FLI1 type 1 translocation was detected by FISH.

In view of the striking rarity of RES, the standard treatment for this tumor remains absence and mostly referred to clinical regimens of osseous Ewing's sarcoma (ESB). Currently, radical nephrectomy followed by chemotherapy was commendatory. Surgical intervention may incorporate with cavotomy in the presence of venous tumor thrombus, which was frequently reported in RES and was stated to be associated with the presence of pulmonary metastasis $[11,12]$. Chemotherapy was recognized to be beneficial to RES survival [13]. The commonly used chemotherapeutic regimes are comprised of multiple agents including vincristine, ifosfamide, doxorubicin, etoposide, cyclophosphamide, etoposide or actinomycin D. In the latest years, neoadjuvant chemotherapy following biopsy was acceptable [14], however, its survival benefits need to be verified in the future. Radiation has shown some success as salvage therapy for targeted lesions. However, conflicting views exist surrounding recommending radiotherapy as the primary treatment modality [15]. Given the multiple metastasis and the poor physical condition of this patient, radiation therapy was not recommended by oncologists. Owing to the aggressive adjuvant therapy, the prognosis of localized RES is improved [16], whereas the survival of patients with metastasis at initial diagnosis remains poor [17]. In addition, the newly approved anti-tumor agents, such as the antiangiogenic drug apatinib [18], were claimed to serve as one of the therapeutic options for RES. More studies are needed to validate the benefits of targeted anti-tumor agents including apatinib on the recurrence free and overall survival of RES patient in the future.

Due to its atypical location as stated, the diagnosis of ES/PNET in kidney relies on integrated analysis including histomorphology, multiple immunohistochemical staining and confirmation of molecular-genetic testing. Despite the improved prognosis of this tumor by surgery and adjuvant therapy, optimized and potent therapeutic regimes are still urgently needed.

\section{Abbreviations \\ ES: Ewing sarcoma; PNET: Primitive neuroectodermal tumors; RES: Renal Ewing sarcoma; CT: Computed tomography; IVC: Inferior vena cava; PET-CT: Positron emission tomography-computed tomography; VAC: Vincristine, doxorubicin and cyclophosphamide; IE: Ifosfamide and etoposide; FISH: Fluorescence in situ hybridization testing. \\ Acknowledgements \\ None. \\ Authors' contributions \\ LC: Data analysis, Writing; YX: Data collection; HS: Data collection; HH: Manu- script revision; DZ: Data analysis and manuscript revision. All authors have read and approved the manuscript. \\ Funding \\ None. \\ Availability of data and materials \\ All data and materials in the manuscript will be available from the correspond- ing author for non-commercial purposes}

\section{Ethics approval and consent to participate}

The Institutional Review Board of Yijishan Hospital Ethics Committee approved the present case report.

\section{Consent for publication}

Written informed consent was obtained from the patient for publication of this manuscript.

\section{Competing interests}

The authors report no conflicts of interest in this work.

\section{Author details}

${ }^{1}$ Department of Urology, Anhui Province, The First Affiliated Hospital of Wannan Medical College, Wuhu, People's Republic of China. ${ }^{2}$ Department of Pathology, Anhui Province, The First Affiliated Hospital of Wannan Medical College, Wuhu, People's Republic of China. 
Received: 17 September 2020 Accepted: 4 November 2020

Published online: 11 November 2020

\section{References}

1. Celli R, Cai G. Ewing sarcoma/primitive neuroectodermal tumor of the kidney: a rare and lethal entity. Arch Pathol Lab Med. 2016;140(3):281-5.

2. Seemayer TA, Thelmo WL, Bolande RP, Wiglesworth FW. Peripheral neuroectodermal tumors. Persp Pediatr Pathol. 1975;2:151-72.

3. Zhang S, Li Y, Wang R, Song B. Ewing's sarcoma/primitive neuroectodermal tumor of the kidney: a case report and literature review. Transl Androl Urol. 2019;8(5):562-6.

4. Ellinger J, Bastian PJ, Hauser S, Biermann K, Muller SC. Primitive neuroectodermal tumor: rare, highly aggressive differential diagnosis in urologic malignancies. Urology. 2006;68(2):257-62.

5. Riley DS, Barber MS, Kienle GS, Aronson JK, von Schoen-Angerer T, Tugwell P, Kiene H, Helfand M, Altman DG, Sox H, et al. CARE guidelines for case reports: explanation and elaboration document. J Clin Epidemiol. 2017:89:218-35.

6. Jimenez RE, Folpe AL, Lapham RL, Ro JY, O'Shea PA, Weiss SW, Amin MB. Primary Ewing's sarcoma/primitive neuroectodermal tumor of the kidney: a clinicopathologic and immunohistochemical analysis of 11 cases. Am J Surg Pathol. 2002;26(3):320-7.

7. Risi E, lacovelli R, Altavilla A, Alesini D, Palazzo A, Mosillo C, Trenta P, Cortesi E. Clinical and pathological features of primary neuroectodermal tumor/ Ewing sarcoma of the kidney. Urology. 2013;82(2):382-6.

8. Parham DM, Roloson GJ, Feely M, Green DM, Bridge JA, Beckwith JB. Primary malignant neuroepithelial tumors of the kidney: a clinicopathologic analysis of 146 adult and pediatric cases from the National Wilms'Tumor Study Group Pathology Center. Am J Surg Pathol. 2001;25(2):133-46.

9. Folpe AL, Goldblum JR, Rubin BP, Shehata BM, Liu W, Dei Tos AP, Weiss SW. Morphologic and immunophenotypic diversity in Ewing family tumors: a study of 66 genetically confirmed cases. Am J Surg Pathol. 2005:29(8):1025-33.
10. Yang Y, Zhang L, Wei Y, Wang H, Xiong W, Chen Z, Hes O, Zheng J. Detection of EWSR1 translocation with nuclear extraction-based fluorescence in situ hybridization for diagnosis of Ewing's sarcoma/primitive neuroectodermal tumor. Anal Quant Cytol Histol. 2007;29(4):221-30.

11. Karpate A, Menon S, Basak R, Yuvaraja TB, Tongaonkar HB, Desai SB. Ewing sarcoma/primitive neuroectodermal tumor of the kidney: clinicopathologic analysis of 34 cases. Ann Diagn Pathol. 2012;16(4):267-74.

12. Fergany AF, Dhar N, Budd GT, Skacel M, Garcia JA. Primary extraosseous ewing sarcoma of the kidney with level III inferior vena cava thrombus. Clin Genitourin Cancer. 2009;7(3):E95-97.

13. Miser JS, Kinsella TJ, Triche TJ, Steis R, Tsokos M, Wesley R, Horvath K, Belasco J, Longo DL, Glatstein E, et al. Treatment of peripheral neuroepithelioma in children and young adults. J Clin Oncol. 1987;5(11):1752-8.

14. Rowe RG, Thomas DG, Schuetze SM, Hafez KS, Lawlor ER, Chugh R. Ewing sarcoma of the kidney: case series and literature review of an often overlooked entity in the diagnosis of primary renal tumors. Urology. 2013;81(2):347-53.

15. Thomas JC, Sebek BA, Krishnamurthi V. Primitive neuroectodermal tumor of the kidney with inferior vena cava and atrial tumor thrombus. J Urol. 2002;168(4 Pt 1):1486-7.

16. Grier HE, Krailo MD, Tarbell NJ, Link MP, Fryer CJH, Pritchard DJ, Gebhardt MC, Dickman PS, Perlman EJ, Meyers PA, et al. Addition of ifosfamide and etoposide to standard chemotherapy for Ewing's sarcoma and primitive neuroectodermal tumor of bone. N Engl J Med. 2003;348(8):694-701.

17. Ladenstein R, Potschger U, Le Deley MC, Whelan J, Paulussen M, Oberlin $\mathrm{O}$, van den Berg H, Dirksen U, Hjorth L, Michon J, et al. Primary disseminated multifocal Ewing sarcoma: results of the Euro-EWING 99 trial. J Clin Oncol. 2010;28(20):3284-91.

18. Zhao Y, Chen Y, Cheng K, Li ZP, Zeng H, Liu JY. Renal Ewing sarcoma treated with apatinib. Anticancer Drugs. 2018;29(7):702-4.

\section{Publisher's Note}

Springer Nature remains neutral with regard to jurisdictional claims in published maps and institutional affiliations.
Ready to submit your research? Choose BMC and benefit from:

- fast, convenient online submission

- thorough peer review by experienced researchers in your field

- rapid publication on acceptance

- support for research data, including large and complex data types

- gold Open Access which fosters wider collaboration and increased citations

- maximum visibility for your research: over $100 \mathrm{M}$ website views per year

At BMC, research is always in progress.

Learn more biomedcentral.com/submissions 